\title{
REPLICATION STUDY OF GENETIC LOCI INFLUENCING AGE AT MENOPAUSE IN SOUTHERN CHINESE WOMEN
}

Hang Wun Raymond $\mathrm{Li}^{1}$, Ching Lung Cheung ${ }^{2,3}$, Kathryn C.B. Tan ${ }^{2}$, Pak Chung Sham ${ }^{3}$, Annie W.C. Kung ${ }^{2}$, Grace Wai King Tang ${ }^{1}$

${ }^{1}$ University of Hong Kong, Department of Obstetrics and Gynaecology, Hong Kong, Hong Kong, China

${ }^{2}$ University of Hong Kong, Department of Medicine, Hong Kong, Hong Kong, China

${ }^{3}$ University of Hong Kong, Centre for Genomic Sciences, Hong Kong, Hong Kong, China

INTRODUCTION: Age at menopause is a highly heritable trait. Previous genome-wide meta-analysis in European and Northern Chinese identified 26 loci underlying age at menopause. OBJECTIVES: To validate these 26 genetic loci in Southern Chinese women. AIMS: To study genetic factors which may influence age at menopause in Southern Chinese. METHODS: This study was performed on 653 women who participated in the Hong Kong Osteoporosis Study, whose age at menopause was available. These women consented to have blood taken and archived for genotyping. DNA was extracted from the buffy coat, and genotyping was performed using Sequenom iPLEX. Age at menopause was recorded using a structured questionnaire. In this study, 60 subjects with early menopause (defined as age at menopause <45 years) were compared to 397 subjects with age at menopause $\geq 50$ years. The associations of 26 SNPs, previously reported in Europeans and northern Chinese, with age at menopause (continuous variable) and early menopause (dichotomized variable) were examined using linear regression and logistic regression, respectively. RESULTS: Among those genotyped loci, rs 10183486, rs11668344, and rs365132 showed significant replication with age at menopause with an effect size of the minor allele being $-0.025(P=0.036),-0.016(P=0.019)$, and 0.009 ( $P$ $=0.017$ ), respectively. Similarly, these SNPs (rs10183486, rs11668344, and rs365132) were also associated with risk of early menopause with the odds ratio of the minor allele being $2.39(P=0.047), 1.88(P=0.029)$, and $0.711(P=0.043)$, respectively. In addition, rs10852344 also showed significant replication with the risk of early menopause with an odds ratio of $0.59(P=0.029)$. CONCLUSIONS: The current study provides independent evidence that rs10183486, rs11668344, rs365132, and possibly rs10852344 are determinants of age at menopause in southern Chinese. The mechanisms of how these associated genes act towards occurrence of menopause warrants further investigation. 